\title{
Applying multi-phase particle swarm optimization to solve bulk cargo port scheduling problem
}

\author{
Tang, M. ${ }^{b, c}$, Gong, D. ${ }^{a, b, c,{ }^{*}}$, Liu, S. ${ }^{b, c}$, Zhang, H. ${ }^{b, c}$ \\ ${ }^{a}$ School of Economics \& Management, Tsinghua University, China \\ ${ }^{\mathrm{b}}$ School of Economics \& Management, Beijing Jiaotong University, China \\ 'International Center for Informatics Research, Beijing Jiaotong University, China
}

\begin{abstract}
A B S T R A C T
Factors related to bulk cargo port scheduling are very complex and peculiar. Changes in the factors will affect the reusability of a model, so establishing a reliable scheduling model for bulk cargo ports is particularly important. This paper sorts the factors affecting bulk cargo port scheduling, such as the number of vessels, the number of berths, vessel-berthing constraints (basic factors), the service priority, and the makespan (special factors), and then establishes the non-deterministic polynomial (NP) model, which aims to minimize the total service time and makespan. Lastly, it solves the model based on the multi-phase particle swarm optimization (MPPSO) algorithm and Matlab. Some important conclusions are obtained. (1) For the model neglecting priority, the total service time is the smallest, whereas the maximum waiting time and maximum operating time are relatively large, and the makespan is the latest. (2) For the model considering priority, the total service time is relatively large, whereas the maximum waiting time and maximum operating time are relatively small, and the makespan is relatively early. (3) For the model considering the makespan, the total service time is the mostlargest, whereas the maximum waiting time and especially the maximum operating time are the smallest, and the makespan is the earliest. We can choose different models according to different situations in bulk cargo port scheduling.
\end{abstract}

\section{ARTICLE INFO}

Keywords:

Bulk cargo

Scheduling

Priority

Makespan

Multi-phase particle swarm optimization (MPPSO)

*Corresponding author: gongtuipigua@163.com (Gong, D.)

Article history:

Received 7 October 2016

Revised 9 November 2016

Accepted 10 November 2016

\section{References}

[1] Cheong, C.Y., Tan, K.C., Liu, D.K., Lin, C.J. (2010). Multi-objective and prioritized berth allocation in container ports, Annals of Operations Research November, Vol. 180, No. 1, 63-103, doi: 10.1007/s10479-008-0493-0.

[2] Conway, R.W., Maxwell, W.L., Miller, L.W. (2003). Theory of scheduling, Dover publication, New York, USA.

[3] Gantt, H. (1919). Organization for Work, Allen and Unwin, London, UK.

[4] Xu, D., Li, C.L., Leung, J.Y.-T. (2012). Berth allocation with time-dependent physical limitations on vessels, European Journal of Operational Research, Vol. 216, No. 1, 47-56, doi: 10.1016/j.ejor.2011.07.012.

[5] Raa, B., Dullaert, W., Van Schaeren, R. (2011). An enriched model for the integrated berth allocation and quay crane assignment problem, Expert Systems with Applications, Vol. 38, No. 11, 14136-14147, doi: 10.1016/i.eswa. 2011.04.224.

[6] Imai, A., Nishimura, E., Papadimitriou, S. (2001). The dynamic berth allocation problem for a container port, Transportation Research Part B: Methodological, Vol. 35, No. 4, 401-417, doi: 10.1016/S0191-2615(99)00057-0.

[7] Guan, Y., Xiao, W.Q., Cheung, R.K., Li, C.L. (2002). A multiprocessor task scheduling model for berth allocation: Heuristic and worst-case analysis, Operations Research Letters, Vol. 30, No. 5, 343-350, doi: 10.1016/S01676377(02) 00147-5.

[8] Arango, C., Cortés, P., Muñuzuri, J., Onieva, L. (2011). Berth allocation planning in Seville inland port by simulation and optimization, Advanced Engineering Informatics, Vol. 25, No. 3, 452-461, doi: 10.1016/i.aei.2011.05.001. 
[9] Galzina, V., Lujić, R., Šarić, T. (2012). Adaptive fuzzy particle swarm optimization for flow-shop scheduling problem, Technical Gazette - Tehnički vjesnik, Vol. 19, No. 1,151-157.

[10] Meisel, F., Bierwirth, C. (2009). Heuristics for the integration of crane productivity in the berth allocation problem, Transportation Research Part E: Logistics and Transportation Review, Vol. 45, No. 1, 196-209, doi: 10.1016/i.tre.2008.03.001.

[11] Golias, M.M., Saharidis, G.K., Boile, M., Theofanis S., Ierapetritou M.G. (2009). The berth allocation problem: Optimizing vessel arrival time, Maritime Economics \& Logistics, Vol. 11, No. 4, 358-377, doi: 10.1057/mel.2009.12.

[12] Türkoğulları, Y.B., Taşkın, Z.C., Aras, N., Altınel, İ.K. (2014). Optimal berth allocation and time-invariant quay crane assignment in container terminals, European Journal of Operational Research, Vol. 235, No. 1, 88-101, doi: 10.1016/j.ejor.2013.10.015.

[13] Imai, A., Sun, X., Nishimura, E., Papadimitriou, S. (2005). Berth allocation in a container port: Using a continuous location space approach, Transportation Research Part B: Methodological, Vol. 39, No. 3, 199-221, doi: 10.1016/ j.trb.2004.04.004.

[14] Legato, P., Mazza, R.M. (2001). Berth planning and resources optimization at a container terminal via discrete event simulation, European Journal of Operational Research, Vol. 133, No. 3, 537-547, doi: 10.1016/S03772217(00)00200-9.

[15] Robenek, T., Umang, N., Bierlaire, M., Ropke, S. (2014). A branch-and-price algorithm to solve the integrated berth allocation and yard assignment problem in bulk ports, European Journal of Operational Research, Vol. 235, No. 2, 399-411, doi: 10.1016/i.ejor.2013.08.015.

[16] Nishimura, E., Imai, A., Papadimitriou, S. (2001). Berth allocation planning in the public berth system by genetic algorithms, European Journal of Operational Research, Vol. 131, No. 2, 282-292, doi: 10.1016/S03772217(00)00128-4.

[17] Imai, A., Zhang, J.T., Nishimura, E., Papadimitriou, S. (2007). The berth allocation problem with service time and delay time objectives, Maritime Economics \& Logistics, Vol. 9, No. 4, 269-290, doi: 10.1057/palgrave.mel. 9100186.

[18] Fu, Y.M., Diabat, A., Tsai, I-T. (2014). A multi-vessel quay crane assignment and scheduling problem: Formulation and heuristic solution approach, Expert Systems with Applications, Vol. 41, No. 15, 6959-6965, doi: 10.1016/i.eswa. 2014.05.002.

[19] Wang, J.F., Kang, W.L., Zhao, J.L., Chu, K.Y. (2016). A simulation approach to the process planning problem using a modified particle swarm optimization, Advances in Production Engineering \& Management, Vol. 11, No. 2, 77-92, doi: 10.14743/apem2016.2.211.

[20] Mocnik, D., Paulic, M., Klancnik, S., Balic, J. (2014) Prediction of dimensional deviation of workpiece using regression, ANN and PSO models in turning operation, Technical Gazette - Tehnički vjesnik, Vol. 21, No. 1, 55-62.

[21] Ting, C.J., Wu, K.C., Chou, H. (2014). Particle swarm optimization algorithm for the berth allocation problem, Expert Systems with Applications, Vol. 41, No. 4 (Part 1), 1543-1550, doi: 10.1016/j.eswa.2013.08.051. 


\section{APEM}

\title{
Uporaba več stopenjske optimizacije $z$ rojem delcev za rešitev problema razvrščanja razsutih ladijskih tovorov
}

\author{
Tang, M. ${ }^{b, c}$, Gong, D. ${ }^{a, b, c,{ }^{*}}$, Liu, S. ${ }^{b, c}$, Zhang, H. ${ }^{b, c}$ \\ ${ }^{a}$ School of Economics \& Management, Tsinghua University, China \\ ${ }^{b}$ School of Economics \& Management, Beijing Jiaotong University, China \\ 'International Center for Informatics Research, Beijing Jiaotong University, China
}

\begin{abstract}
POVZETEK
Faktorji, ki vplivajo na razvrščanje razsutih tovorov v pristaniščih so zelo specifični in zapleteni. Ker spremembe faktorjev vplivajo na uporabnost modela, je vzpostavitev zanesljivega modela posebej pomembna. V prispevku so vplivni faktorji razvrščeni na število plovil, število privezov, omejitve priveza na plovilo (osnovni faktor), prednost raztovarjanja in pretočni čas (poseben faktor). Na podlagi faktorjev je zgrajen ne deterministični polinomski model, s katerim poiščemo najmanjši skupni čas priveza in najmanjši pretočni čas. Model rešimo z več stopenjskim algoritmom roja delcev v programu Matlab. Pomembnejši zaključki so: (1) Brez upoštevanja prednosti je skupni čas priveza najkrajši, najdaljši čas čakanja in najdaljši čas obratovanja pa sta razmeroma dolga. Pretočni čas je najdaljši. (2) Z upoštevanjem prednosti je skupni čas priveza razmeroma dolg, najdaljši čas čakanja in najdaljši čas obratovanja pa sta razmeroma kratka, enako kot pretočni čas. (3) Z upoštevanjem faktorja pretočnega časa je skupni čas priveza najdaljši. Najdaljši čas čakanja in posebej najdaljši čas obratovanja sta najkrajša. Pretočni čas je najkrajši. V različnih situacijah lahko izberemo različne modele razvrščanja tovorov v pristaniščih.
\end{abstract}

(C) 2016 PEI, University of Maribor. All rights reserved.

\section{PODATKI O ČLANKU}

Ključne besede:

Velik tovor

Razvrščanje

Prednosti

Več stopenjska optimizacija z

rojem delcev

*Kontaktna oseba:

gongtuipigua@163.com

(Gong, D.)

Zgodovina članka:

Prejet 7. oktobra 2016

Popravljen 9. novembra 2016

Sprejet 10. novembra 2016 